\title{
Feedback linearization and stabilization of second-order nonholonomic chained systems
}

\author{
S. S. GE* ZHENDONG SUN, T. H. LEE and MARK W. SPONG \\ Department of Electrical Engineering \\ Coordinated Science Lab \\ National University of Singapore \\ Singapore 117576 \\ University of Illinois at Urbana-Champaign \\ Urbana, IL 61801, USA
}

This paper presents a theoretical framework for nonregular feedback linearization and stabilization of second-order nonholonomic chained systems. By giving a new criterion for the problem of nonsmooth nonregular feedback linearization, it is proved that second-order chained systems are nonregular static state feedback linearizable. A discontinuous control law is obtained based on linear system theory and the inversion technique. The design mechanism is generalised to higher-order nonholonomic chained systems. Simulation studies are carried out to show the effectiveness of the approach.

\section{Introduction}

The problem of controlling nonholonomic systems has attracted much attention in the last decade. These studies were primarily limited to first-order nonholonomic systems which undergo non-integrable kinematic constraints (Kolmanovsky and McClamroch 1995, Canudas de Wit, Siciliano, Bastin 1996, Luo and Tsiotras 2000). There are only a few publications which address the problem of controlling nonholonomic systems satisfying non-integrable acceleration or dynamic relationships. This class of systems is referred to as second-order or high-order nonholonomic systems (Oriolo and Nakamura 1991, Laiou and Astolfi 1999).

Second-order and high-order nonholonomic systems arise very often in the study of mechanical systems. Typical examples include redundant manipulators (De Luca, Mattone and Oriolo 1996, 1998) and underactuated systems (Oriolo and Nakamura 1991, Egeland and Berglund 1994, Seto and Baillieul 1994, Spong 1995, Reyhanoglu

* To whom all correspondence should be addressed. Tel. (+65) 8746821; Fax. (+65) 7791103; E-mail: elegesz@nus.edu.sg. 
et al. 1999). Although the dynamics are well understood and many techniques have been investigated for these systems, controller design of these systems remains a challenging problem. Attempts were made to stabilize second-order or high-order nonholonomic systems recently. Among them, the most notable contribution is due to Laiou and Astolfi (1999), who successfully generalise the idea proposed in Astolfi (1996) to a class of high-order nonholonomic systems with two inputs. In this paper, a class of discontinuous state feedback laws was presented to exponentially stabilize the systems. Some research works have also been presented for specific classes of systems in the literature. Controllability and stabilizability have been investigated for a special class of underactuated systems in Reyhanoglu et al. (1999). In Su and Stepanenko (1999), variable structure control has been applied to the control of underactuated robots. Homogeneous design technique is exploited in M'Closkey and Morin (1998) to obtain time-varying homogeneous feedback for a class of nonlinear systems with drifts which includes some underactuated mechanical systems. Based on a novel design technique proposed in Sordalen (1993), a time-varying controller was obtained for a second-order chained form in Egeland and Berglund (1994) to achieve asymptotic stability with exponential convergence.

It has been proven that several classes of second-order nonholonomic systems are transformable into the following chained form by smooth state and input transformations

$$
\left\{\begin{array}{l}
\ddot{y}_{1}=u_{1} \\
\ddot{y}_{2}=u_{2} \\
\ddot{y}_{3}=y_{2} u_{1}
\end{array}\right.
$$

Typical examples include a three-link planar manipulator with the third joint unactuated (Arai, Tanie and Shiroma 1997), an (underactuated) underwater vehicle with nonholonomic acceleration constraints (Egeland and Berglund 1994), an underactuated surface vessel with two independent propellers (Reyhanoglu et al. 1999, Wichlund, Sordalen and Egeland 1995), and a kinematic redundant planar PPR manipulator with all joints passive and steered by the forces imposed at the end-effector (De Luca, Mattone and Oriolo 1996, 1998).

In this paper, the problem of feedback stabilization is addressed for second-order 
nonholonomic chained system (1) in the framework of nonregular feedback linearization. In Section 2, a new sufficient condition for nonsmooth nonregular feedback linearization is presented, and the second-order nonholonomic chained systems are then proved to be nonregular feedback linearizable. In Section 3, a discontinuous feedback control law is designed based on linear system theory and the inversion technique. The design mechanism is extended to general high-order nonholonomic chained systems in Section 4. The effectiveness of the proposed control law is illustrated through simulation study in Section 5. The last section presents concluding remarks.

\section{Mathematical preliminaries}

Feedback linearization is a standard technique for control of many nonlinear systems, for example, robotic systems (Spong and Vidyasagar 1989, Ge, Lee and Harris 1998), motor drives (Taylor 1994, Chiasson 1998) and among others. For nonholonomic systems, however, it is known that they are not regular static state feedback linearizable. Accordingly, the classical approach of feedback linearization cannot be applied. To cope with this difficulty, more general classes of feedback should be introduced. One candidate is dynamic feedback, but it means that the original systems must be augmented to include additional dynamics. Another possible choice is nonregular static state feedback, which does not introduce additional dynamics. It happens that the latter is applicable to the second-order nonholonomic chained systems as illustrated below.

Consider the multi-input affine nonlinear system given by

$$
\dot{x}=f(x)+\sum_{i=1}^{m} u_{i} g_{i}(x)=f(x)+G(x) u
$$

where $x \in \Re^{n}$ are the states, $u \in \Re^{m}$ are the inputs, entries of $f(x)$ and $G(x)$ are analytic functions of $x$, and $\operatorname{rank} G(x)=m, \forall x \in \Re^{n}$.

Nonlinear control system (2) is said to be (nonsmooth) nonregular (static state) 
feedback linearizable, if there exist a discontinuous state transformation

$$
z=T(x), z \in \Re^{n}
$$

and a nonregular state feedback

$$
u(t)=\alpha(x)+\beta(x) v(t), v \in \Re^{m_{0}}, m_{0} \leq m
$$

such that the transformed system with state $z$ and input $v$ reads as a controllable linear system.

Note that state transformation (3) is not necessarily of diffeomorphism as required in Definition 2.1 of Sun and Xia (1997). However, its inverse transformation should be well defined everywhere except for on a lower-dimensional submanifold of $\Re^{n}$. The term discontinuous is used to denote functions which are unbounded and hence undefined on a certain set (Astolfi 1996).

Remark 1. The problem of linearizing a nonlinear system via nonregular state feedback arises naturally and was proposed as an open problem in Charlet, Levine and Marino (1989). Though the concept is rather simple, it is very difficult to judge whether a system is nonregular feedback linearizable. Even if the answer is affirmative, it is also very hard to find a linearizing feedback and the corresponding state coordinate transformation. Until now, only some preliminary results were reported in Sun and Xia (1997) and no extensive studies have been found in the literature.

The following lemma presents a new criterion for nonregular static state feedback linearizability.

Lemma 1. For a two-input affine nonlinear system

$$
\dot{x}=f(x)+g_{1}(x) u_{1}+g_{2}(x) u_{2}, \quad x \in \Re^{n}
$$

suppose there exist a natural number $\mu \geq 1$ and a set of integer $1 \leq \kappa_{1}<\kappa_{2}<\cdots<$ $\kappa_{\mu} \leq n-1$, such that the nested distributions defined by

$$
\begin{aligned}
& \Delta_{0}=\operatorname{span}\left\{g_{2}\right\} \\
& \Delta_{i}=\Delta_{i-1}+a d_{g_{1}} \Delta_{i-1}, i=\kappa_{1}, \cdots, \kappa_{\mu-1} \\
& \Delta_{\kappa_{\mu}}=\Delta_{\kappa_{\mu}-1}+\operatorname{span}\left\{g_{1}\right\} \\
& \Delta_{i}=\Delta_{i-1}+a d_{f} \Delta_{i-1}, i \geq 1, i \neq \kappa_{1}, \cdots, \kappa_{\mu}
\end{aligned}
$$


satisfy

(i) $\Delta_{i}$ is involutive and of constant rank for $0 \leq i \leq n-1$;

(ii) $\operatorname{rank} \Delta_{n-1}=n$;

(iii) $\left[f, \Delta_{\kappa_{j}-1}\right] \in \Delta_{\kappa_{j}-1}$ for $j=1, \cdots, \mu$; and

(iv) $\left[g_{1}, \Delta_{i}\right] \in \Delta_{i+1} \quad$ for $0 \leq i \leq n-3$

then system (5) is nonregular feedback linearizable.

Proof. By the Frobenius Theorem, there exist real-valued functions $\phi(x)$ and $h(x)$ : $\Re^{n} \rightarrow \Re$, such that

$$
d \phi \perp \Delta_{\kappa_{\mu}-2}, d \phi \not \perp \Delta_{\kappa_{\mu}-1}, d h \perp \Delta_{n-2}, \quad \text { and } L_{a d_{f}^{n-\kappa_{\mu}-1} g_{1}} h=1
$$

Let

$$
u_{1}=\phi(x), \quad f_{0}(x)=f(x)+\phi(x) g_{1}(x)
$$

System (5) can be rewritten as

$$
\dot{x}=f_{0}(x)+u_{2} g_{2}(x)
$$

It follows from (6) that, if $L_{f}^{i} g_{1} \in \Delta_{j}$ for some $i$ and $j$, then $L_{f}^{i+1} g_{2} \in \Delta_{j}$. This condition is repeatedly used in the following derivation.

Compute the following quantities

$$
L_{g_{2}} h=0, \quad L_{f_{0}} h=L_{f} h+\phi L_{g_{1}} h=L_{f} h
$$

The derivative of $h$ along system (8) is given by

$$
\frac{d h}{d t}=L_{f_{0}} h+\left(L_{g_{2}} h\right) u_{2}=L_{f} h
$$

Its higher derivatives are given by

$$
\begin{aligned}
\frac{d^{2} h}{d t^{2}} & =L_{f_{0}}^{2} h+\left(L_{g_{2}} L_{f_{0}} h\right) u_{2}=L_{f}^{2} h \\
& \vdots \\
\frac{d^{n-\kappa_{\mu}-1} h}{d t^{n-\kappa_{\mu}-1}} & =L_{f_{0}}^{n-\kappa_{\mu}-1} h+\left(L_{g_{2}} L_{f_{0}}^{n-\kappa_{\mu}-1} h\right) u_{2}=L_{f}^{n-\kappa_{\mu}-2} h \\
\frac{d^{n-\kappa_{\mu}} h}{d t^{n-\kappa_{\mu}}} & =L_{f_{0}}^{n-\kappa_{\mu}} h+\left(L_{g_{2}} L_{f_{0}}^{n-\kappa_{\mu}-1} h\right) u_{2}=L_{f}^{n-\kappa_{\mu}} h+\phi L_{a d_{f}^{n-\kappa_{\mu}-1} g_{1}} h=L_{f}^{n-\kappa_{\mu}} h+\phi
\end{aligned}
$$


From assumptions (iii) (iv) and conditions (7), it follows that

$$
\begin{aligned}
L_{g_{2}} L_{f}^{i} h & =0, i=0,1, \cdots \\
L_{g_{2}} L_{f}^{i} \phi & =0, i=0,1, \cdots \\
L_{g_{2}} L_{f}^{j} L_{g_{1}} L_{f}^{\kappa_{l}-\kappa_{l-1}-1} \cdots L_{g_{1}} L_{f}^{\kappa_{\mu+1}-\kappa_{\mu}-1} h & =0, l=\mu, \cdots, 2, j=0,1, \cdots, \xi_{l-1} \\
L_{g_{2}} L_{f}^{j} L_{g_{1}} L_{f}^{\kappa_{l}-\kappa_{l-1}-1} \cdots L_{g_{1}} L_{f}^{\kappa_{\mu}-\kappa_{\mu-1}-1} L_{g_{1}} \phi & =0, l=\mu, \cdots, 2, j=0,1, \cdots, \xi_{l-1} \\
L_{g_{2}} L_{f}^{\kappa_{1}-1} L_{g_{1}} L_{f}^{\kappa_{2}-\kappa_{1}-1} \cdots L_{g_{1}} L_{f}^{\kappa_{\mu}-\kappa_{\mu-1}-1} L_{g_{1}} \phi & \neq 0
\end{aligned}
$$

where $\kappa_{0}=1, \kappa_{\mu+1}=n+1, \xi_{l}=\kappa_{l}-\kappa_{l-1}-1, l=1, \cdots, \mu$.

Keeping these in mind, we can further compute higher order derivatives of $h$ along system (8) as follows

$$
\begin{aligned}
\frac{d^{n-\kappa_{\mu}+1} h}{d t^{n-\kappa_{\mu}+1}}= & L_{f_{0}}^{n-\kappa_{\mu}+1} h+\left(L_{g_{2}} L_{f_{0}}^{n-\kappa_{\mu}} h\right) u_{2}=L_{f_{0}} L_{f}^{n-\kappa_{\mu}} h+L_{f_{0}} \phi \\
& \vdots \\
\frac{d^{n-2} h}{d t^{n-2}}= & L_{f_{0}}^{n-2} h+\left(L_{g_{2}} L_{f_{0}}^{n-3} h\right) u_{2}=L_{f_{0}}^{\kappa_{\mu}} L_{f}^{n-\kappa_{\mu}} h+L_{f_{0}}^{\kappa_{\mu} \phi} \\
\frac{d^{n-1} h}{d t^{n-1}}= & L_{f_{0}}^{n-1} h+\left(L_{g_{2}} L_{f_{0}}^{n-2} h\right) u_{2}=L_{f_{0}}^{\kappa_{\mu+1}} L_{f}^{n-\kappa_{\mu}} h+L_{f_{0}}^{\kappa_{\mu+1} \phi} \\
& +\left(L_{g_{2}} L_{f}^{\kappa_{1}-1} L_{g_{1}} L_{f}^{\kappa_{2}-\kappa_{1}-1} \cdots L_{g_{1}} L_{f}^{\kappa_{\mu}-\kappa_{\mu-1}-1} L_{g_{1}} \phi\right) u_{2}
\end{aligned}
$$

Define new coordinates $z$ and new input $v$ respectively as follows

$$
\begin{aligned}
& z=\left[h, L_{f} h, \cdots, L_{f}^{n-\kappa_{\mu}} h, L_{f_{0}} L_{f}^{n-\kappa_{\mu}} h+L_{f_{0}} \phi, \cdots, L_{f_{0}}^{\kappa_{\mu+1}} L_{f}^{n-\kappa_{\mu}} h+L_{f_{0}}^{\kappa_{\mu}} \phi\right]^{T} \\
& v=L_{f_{0}}^{\kappa_{\mu+1}} L_{f}^{n-\kappa_{\mu}} h+L_{f_{0}}^{\kappa_{\mu+1}} \phi+\left(L_{g_{2}} L_{f}^{\kappa_{1}-1} L_{g_{1}} L_{f}^{\kappa_{2}-\kappa_{1}-1} \cdots L_{g_{1}} L_{f}^{\kappa_{\mu}-\kappa_{\mu-1}-1} L_{g_{1}} \phi\right) u_{2}
\end{aligned}
$$

The state space description of system (8) in the $z$ coordinates is then given by

$$
\dot{z}=\left[z_{2}, z_{3}, \cdots, z_{n}, v\right]^{T}
$$

which is exactly the single-input Brunovsky canonical system.

The above analysis shows that, under the state feedback

$$
\begin{aligned}
& u_{1}=\phi(x) \\
& u_{2}=\left(L_{g_{2}} L_{f}^{\kappa_{1}-1} L_{g_{1}} L_{f}^{\kappa_{2}-\kappa_{1}-1} \cdots L_{g_{1}} L_{f}^{\kappa_{\mu}-\kappa_{\mu-1}-1} L_{g_{1}} \phi\right)^{-1}\left(v-L_{f_{0}}^{\kappa_{\mu+1}} L_{f}^{n-\kappa_{\mu}} h+L_{f_{0}}^{\kappa_{\mu+1}} \phi\right)
\end{aligned}
$$


and the coordinate transformation (10), system (5) changes into the single-input controllable system (12). Because the input channel $u_{1}$ is pure state feedback, the overall input transformation (13) is nonregular. $\diamond$

Remark 2. Note that the above analysis is essentially nonsmooth analysis in that the functions involved are not limited to continuous functions. State transformation (10) is not necessarily of diffeomorphism, whereas input transformations (11) and (13) may not be well defined on a lower-dimensional submanifold of $\Re^{n}$.

As a direct application of Lemma 1, we claim that the high-order nonlinear system

$$
\left\{\begin{array}{l}
y_{1}{ }^{\left(r_{1}\right)}=\xi_{1}\left(x^{1}, u_{1}\right) \\
y_{2}{ }^{\left(r_{2}\right)}=\xi_{2}\left(x, u_{2}\right) \\
y_{i}{ }^{\left(r_{i}\right)}=\xi_{i}\left(x^{1}, y_{i-1}, x^{i}, \cdots, x^{l}, u_{1}\right), i=3, \cdots, l
\end{array}\right.
$$

is nonregular feedback linearizable, where $l \geq 3, r_{i} \geq 1, x^{i}=\left[y_{i}, \cdots, y_{i}^{\left(r_{i}-1\right)}\right]^{T}$, $i=1, \cdots, l, x=\left[x^{1^{T}}, \cdots, x^{l^{T}}\right]^{T}$, and $\xi_{i}, i=1, \cdots, l$ are analytic functions vanishing at the origin with

$$
\frac{\partial \xi_{1}}{\partial u_{1}} \neq 0, \frac{\partial \xi_{2}}{\partial u_{2}} \neq 0, \frac{\partial^{2} \xi_{i}}{\partial y_{i-1} \partial u_{1}} \neq 0, i=3, \cdots, l
$$

The linearizing output $h(x)$ and the real-valued function $\phi(x)$ in the proof of Lemma 1 could be explicitly constructed, say

$$
\begin{aligned}
& h(x)=x_{1} \\
& \phi(x)=\phi_{1}\left(y_{1}, \cdots, y_{1}^{\left(r_{1}\right)}, y_{l}\right) \text { with } \frac{\partial \phi_{1}}{\partial y_{l}} \neq 0
\end{aligned}
$$

Note that the above model includes the high order single chained systems

$$
\left\{\begin{array}{l}
y_{1}^{\left(r_{1}\right)}=u_{1} \\
y_{2}^{\left(r_{2}\right)}=u_{2} \\
y_{3}^{\left(r_{3}\right)}=y_{2} u_{1} \\
\vdots \\
y_{l}^{\left(r_{l}\right)}=y_{l-1} u_{1}
\end{array}\right.
$$

as special cases. In general, system (14) and the generalised chained system (1) in Laiou and Astolfi (1999) are not special cases of each other. For example, the simple 
system

$$
\left\{\begin{array}{l}
\ddot{y}_{1}=u_{1} \\
\ddot{y}_{2}=u_{2} \\
\ddot{y}_{3}=y_{2} u_{1}+y_{3}^{2} u_{1}
\end{array}\right.
$$

is in form (14) but is not in form (1) of Laiou and Astolfi (1999).

Remark 3. As the restriction imposed on $\phi(x)$ by (15) is not very restrictive, we can fully explore the freedom in controller design in practice. Different choices of $\phi(x)$ will result in different linearizing feedback transformations.

\section{Controller design}

For second-order nonholonomic chained system (1), a stabilizing strategy in the framework of nonregular feedback linearization is proposed in this section. The resulting control laws render the system exponentially convergent to the origin.

Let $x=\left[x_{1}, \cdots, x_{6}\right]^{T}=\left[y_{1}, \dot{y}_{1}, y_{2}, \dot{y}_{2}, y_{3}, \dot{y}_{3}\right]^{T}$. System (1) can be rewritten in the state space description

$$
\left\{\begin{array}{l}
\dot{x}_{1}=x_{2} \\
\dot{x}_{2}=u_{1} \\
\dot{x}_{3}=x_{4} \\
\dot{x}_{4}=u_{2} \\
\dot{x}_{5}=x_{6} \\
\dot{x}_{6}=x_{3} u_{1}
\end{array}\right.
$$

Let function $\phi(x)=y_{3}{ }^{\frac{1}{3}}=x_{5}{ }^{\frac{1}{3}}$ which satisfies relationship (15). From Lemma 1, we have

$$
u_{1}=x_{5}^{\frac{1}{3}}
$$

Let $h(x)=x_{1}$. From (10), we have the state and input transformations

$$
\begin{aligned}
& z=\left[x_{1}, \quad x_{2}, \quad x_{5} \frac{1}{3}, \quad \frac{1}{3} \frac{x_{6}}{x_{5}{ }^{\frac{2}{3}}}, \quad \frac{1}{3} \frac{x_{3}}{x_{5} \frac{1}{3}}-\frac{2}{9} \frac{x_{6}^{2}}{x_{5}{ }^{\frac{5}{3}}}, \quad \frac{1}{3} \frac{x_{4}}{x_{5} \frac{1}{3}}-\frac{5}{9} \frac{x_{3} x_{6}}{x_{5}{ }^{\frac{4}{3}}}+\frac{10}{27} \frac{x_{6}^{3}}{x_{5}{ }^{\frac{8}{3}}}\right]^{T} \\
& v=-\frac{5}{9} \frac{x_{3}^{2}}{x_{5}}+\frac{50}{27} \frac{x_{3} x_{6}^{2}}{x_{5}{ }^{\frac{7}{3}}}-\frac{2}{3} \frac{x_{4} x_{6}}{x_{5}{ }^{\frac{4}{3}}}-\frac{80}{81} \frac{x_{6}^{4}}{x_{5} \frac{11}{3}}+\frac{1}{3} \frac{u_{2}}{x_{5}{ }^{\frac{1}{3}}}
\end{aligned}
$$


which are discontinuous at the discontinuous surface so defined by

$$
S_{D}=\left\{x: x_{5}=0\right\}=\left\{z: z_{3}=0\right\} .
$$

The inverses of the state and input transformations are

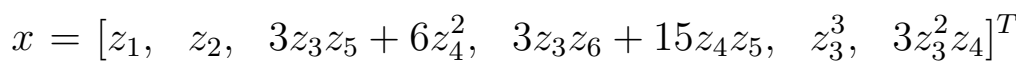

$$
\begin{aligned}
& u=\left[z_{3}, \quad 3 z_{3} v+18 z_{4} z_{6}+45 z_{5}^{2}\right]^{T}
\end{aligned}
$$

which are well-define and smooth on $\Re^{6}$.

The corresponding transformed linear system is of sixth-order Brunovsky canonical form

$$
\dot{z}=\left[\begin{array}{llllll}
z_{2}, & z_{3}, & z_{4}, & z_{5}, & z_{6}, & v
\end{array}\right]^{T}
$$

For linear system (23), controller design can be carried out easily. The desired closed-loop poles can be assigned in different ways. In fact it can be assigned to any arbitrary (symmetric) set. For completeness and simplicity of the paper, let us assign all the poles at $-\lambda$ with $\lambda>0$. Accordingly, the controller is given by

$$
v=-\sum_{i=1}^{6} k_{i} z_{i}
$$

where

$$
k_{1}=\lambda^{6}, k_{2}=6 \lambda^{5}, k_{3}=15 \lambda^{4}, k_{4}=20 \lambda^{3}, k_{5}=15 \lambda^{2}, k_{6}=6 \lambda
$$

Define an unbounded and open subset of $\Re^{6}$ :

$$
\begin{aligned}
D=\left\{x \in \Re^{6}:\right. & x_{5}\left(3 \lambda+x_{6}\right)>0,9 \lambda^{2} x_{5}^{2}+6 \lambda x_{5} x_{6}+3 x_{3} x_{5}^{\frac{4}{3}}-2 x_{6}^{2}>0, \\
& \lambda^{3} x_{5}^{2}+\lambda^{2} x_{5} x_{6}+\lambda x_{3} x_{5}^{\frac{4}{3}}-\frac{2}{3} \lambda x_{6}^{2}+\frac{1}{2} x_{4} x_{5} 5^{\frac{4}{3}}-\frac{5}{9} x_{3} x_{5} \frac{1}{3} x_{6}+\frac{10}{27} \frac{x_{6}^{3}}{x_{5}}>0, \\
& \left(\lambda^{5} x_{1}+5 \lambda^{4} x_{2}\right) x_{5} \frac{5}{3}+10 \lambda^{3} x_{5}^{2}+\frac{10}{3} \lambda^{2} x_{5} x_{6}+\left(\frac{5}{3} \lambda x_{3}+\frac{1}{3} x_{4}\right) x_{5} \frac{4^{3}}{3} \\
& -\frac{10}{9} \lambda x_{6}^{2}-\frac{5}{9} x_{3} x_{5} \frac{1}{3} x_{6}+\frac{10}{27} \frac{x_{6}^{3}}{x_{5}}>0,\left(\lambda^{5} x_{1}+6 \lambda^{4} x_{2}\right) x_{5}^{\frac{5}{3}}+14 \lambda^{3} x_{5}^{2} \\
& \left.+\frac{16}{3} \lambda^{2} x_{5} x_{6}+\left(3 \lambda x_{3}+\frac{2}{3} x_{4}\right) x_{5}^{\frac{4}{3}}-2 \lambda x_{6}^{2}-\frac{10}{9} x_{3} x_{5}{ }^{\frac{1}{3}} x_{6}+\frac{20}{27} \frac{x_{6}^{3}}{x_{5}}<0\right\}
\end{aligned}
$$

The closed-loop stability is summarised in Theorem 1.

Theorem 1. If $x(0) \in D$, then the feedback control law

$$
\left\{\begin{aligned}
u_{1}= & x_{5}^{\frac{1}{3}} \\
u_{2}= & -k_{5} x_{3}-k_{6} x_{4}-3 k_{1} x_{1} x_{5}^{\frac{1}{3}}-3 k_{2} x_{2} x_{5} \frac{1}{3}-3 k_{3} x_{5} \frac{2}{3}-k_{4} \frac{x_{6}}{x_{5}^{\frac{1}{3}}}+\frac{5}{3} \frac{x_{3}^{2}}{x_{5} \frac{2}{3}} \\
& +\frac{5}{3} k_{6} \frac{x_{3} x_{6}}{x_{5}}+2 \frac{x_{4} x_{6}}{x_{5}}+\frac{2}{3} k_{5} \frac{x_{6}^{2}}{x_{5}{ }^{\frac{4}{3}}}-\frac{50}{9} \frac{x_{3} x_{6}^{2}}{x_{5}{ }^{2}}-\frac{10}{9} k_{6} \frac{x_{6}^{3}}{x_{5}{ }^{\frac{7}{3}}}+\frac{80}{27} \frac{x_{6}^{4}}{x_{5} \frac{10}{3}}
\end{aligned}\right.
$$


drives the second-order nonholonomic chained system (16) to the origin at an exponential rate with bounded input.

Proof. Due to the smooth nature of state transformation (21), exponential convergence of $z(t)$ implies exponential convergence of $x(t)$. The linearizing transformations (18) and (19) are well-defined if the trajectory of $z(t)$ do not cross the discontinuous surface (20). As a consequence, if $z_{3}(0) z_{3}(t)>0$ for all $t>0$, then $x(t)$ exponentially converge to the origin.

For the closed-loop system of (23) and (24), it is straightforward to calculate that

$$
z_{3}(t)=e^{-\lambda t} \sum_{i=1}^{6} a_{i} t^{i-1}
$$

where

$$
\begin{aligned}
& a_{1}=z_{3}(0) \\
& a_{2}=\lambda z_{3}(0)+z_{4}(0) \\
& a_{3}=\frac{1}{2}\left(\lambda^{2} z_{3}(0)+2 \lambda z_{4}(0)+z_{5}(0)\right) \\
& a_{4}=\frac{1}{6}\left(\lambda^{3} z_{3}(0)+3 \lambda^{2} z_{4}(0)+3 \lambda z_{5}(0)+z_{6}(0)\right) \\
& a_{5}=-\frac{1}{24}\left(\lambda^{6} z_{1}(0)+6 \lambda^{5} z_{2}(0)+14 \lambda^{4} z_{3}(0)+16 \lambda^{3} z_{4}(0)+9 \lambda^{2} z_{5}(0)+2 \lambda z_{6}(0)\right) \\
& a_{6}=\frac{1}{120}\left(\lambda^{7} z_{1}(0)+5 \lambda^{6} z_{2}(0)+10 \lambda^{5} z_{3}(0)+10 \lambda^{4} z_{4}(0)+5 \lambda^{3} z_{5}(0)+\lambda^{2} z_{6}(0)\right)
\end{aligned}
$$

Note that to guarantee $z_{3}(0) z_{3}(t)>0, \forall t>0$, a sufficient condition is $z_{3}(0) a_{i}>$ $0, \quad i=1,2, \cdots, 6$, which is equivalent to the assumption $x(0) \in D$. Accordingly, state $x(t)$ is bounded and converges to the origin at an exponential rate. By (22), the input $u(t)$ also exponentially converges to zero, and is bounded. $\diamond$

To make chained system (16) globally attractive, we only need to drive any initial configuration into set $D$ by an appropriate control input. For completeness, a switching strategy is outlined below.

Firstly, if $x_{3}(0)=0$, then apply $u_{2}(t)=k_{1}$ in duration $\left[0, t_{1}\right]$ to steer $x_{3}$ away form zero. Secondly, starting from $t_{1}$, apply $u_{1}(t)=-k_{2} \operatorname{sgn}\left(x_{3} x_{6}\right)$ till $x_{5}\left(t_{2}\right) \neq$ 0 and $x_{6}\left(t_{2}\right)=0$. It can be verified that any $k_{2} \neq \operatorname{sgn}\left(x_{3} x_{6}\right) \frac{x_{3}\left(t_{1}\right) x_{6}^{2}(0)}{2 x_{5}(0)}$ with 
$t_{2}=t_{1}+\frac{\left|x_{3}\left(t_{1}\right) x_{6}(0)\right|}{k_{2}}$ will do. Thirdly, apply the finite-time-stabilizing feedback controller (Bhat and Bernstein 1998)

$$
u_{2}(t)=-k_{3}\left[x_{4}^{\frac{1}{3}}+\left(x_{3}+\frac{3}{5} x_{4}^{\frac{5}{3}}\right)^{\frac{1}{5}}\right]
$$

to achieve $x_{3}\left(t_{3}\right)=x_{4}\left(t_{3}\right)=0$ in finite time $t_{3}>t_{2}$. Fourthly, let

$$
u_{1}(t)=-k_{4} \operatorname{sgn}\left(x_{1}-\frac{21}{\lambda^{2}} x_{5}^{\frac{1}{3}}\right)-k_{5} \operatorname{sgn}\left(x_{2}+\frac{6}{\lambda} x_{5}^{\frac{1}{3}}\right)
$$

which steers the state trajectory into $D$ in finite time $t_{4}$. Finally, the feedback control law (25) steers chained system (16) approaching the origin at an exponentially convergent rate. All the $k_{i}$ 's are positive numbers which can be assigned freely.

Denote the respective control laws as

$$
\begin{aligned}
& u^{1}=\left[\begin{array}{l}
0 \\
k_{1}
\end{array}\right] \\
& u^{2}=\left[\begin{array}{c}
-k_{2} \operatorname{sgn}\left(x_{3} x_{6}\right) \\
0
\end{array}\right] \\
& u^{3}=\left[\begin{array}{c}
0 \\
-k_{3}\left[x_{4}^{\frac{1}{3}}+\left(x_{3}+\frac{3}{5} x_{4}^{\frac{5}{3}}\right)^{\frac{1}{5}}\right]
\end{array}\right] \\
& u^{4}=\left[\begin{array}{l}
6 \\
-k_{4} \operatorname{sgn}\left(x_{1}-\frac{1}{\lambda^{2}} x_{5}^{\frac{1}{3}}\right)-k_{5} \operatorname{sgn}\left(x_{2}+\frac{3}{\lambda} x_{5}^{\frac{1}{3}}\right)
\end{array}\right] \\
& u^{5}=\left[\begin{array}{l}
0 \\
-k_{5} x_{3}-k_{6} x_{4}-3 k_{1} x_{1} x_{5}{ }^{\frac{1}{3}}-3 k_{2} x_{2} x_{5} \frac{1}{3}-3 k_{3} x_{5} \frac{2}{3}-k_{4} \frac{x_{6}}{x_{5} \frac{1}{3}}+\frac{5}{3} \frac{x_{3}^{2}}{x_{5}^{\frac{2}{3}}} \\
+\frac{5}{3} k_{6} \frac{x_{3} x_{6}}{x_{5}}+2 \frac{x_{4} x_{6}}{x_{5}}+\frac{2}{3} k_{5} \frac{x_{6}^{2}}{x_{5}}-\frac{50}{9} \frac{x_{3} x_{6}^{2}}{x_{5}{ }^{2}}-\frac{10}{9} k_{6} \frac{x_{6}^{3}}{x_{5} \frac{7}{3}}+\frac{80}{27} \frac{x_{6}^{4}}{x_{5} \frac{10}{3}}
\end{array}\right]
\end{aligned}
$$

Define a logic-based switching controller

$$
u= \begin{cases}u^{5} & \text { if } x \in D \\ u^{4} & \text { if } x \notin D \text { and } x_{3}^{2}+x_{4}^{2}+x_{6}^{2}=0 \neq x_{5} \\ u^{3} & \text { if } x \notin D \text { and }\left(x_{3}^{2}+x_{4}^{2}\right) x_{5} \neq 0=x_{6} \\ u^{2} & \text { if } x \notin D \text { and } x_{3} \neq 0 \text { and }\left(x_{6} \neq 0 \text { or } x_{5}=0\right) \\ u^{1} & \text { else }\end{cases}
$$


This controller steers system (16) globally approaching with an exponential rate of convergence. The total number of switching is less or equal to four.

Remark 4. Note that in the switching strategy (27), the numerically non-detectable criterion $x_{3}^{2}+x_{4}^{2}+x_{6}^{2}=0$ can be relaxed to $x_{3}^{2}+x_{4}^{2}+x_{6}^{2}<\epsilon$ which are numerically detectable, where $\epsilon$ is an appropriate small positive constant. The resulting state configuration (before applying $u^{5}$ ) is

$$
x^{*}=\left[\frac{21}{\lambda^{2}} x_{5}^{\frac{1}{3}}, \frac{-6}{\lambda} x_{5}^{\frac{1}{3}}, x_{3}, x_{4}, x_{5}, x_{6}\right]
$$

with $x_{i}, i=3,4,6$ close to zero. Simple computation shows that, if

$$
6 \lambda \frac{\left|x_{6}\right|}{\left|x_{5}\right|}+5 \lambda^{2} \frac{\left|x_{3}\right|}{x_{5}{ }^{\frac{2}{3}}}+2 \lambda^{3} \frac{\left|x_{4}\right|}{x_{5} \frac{2}{3}}<1
$$

then $x^{*} \in D$. Accordingly, $\epsilon$ can be selected as

$$
\epsilon=\frac{1}{6 \max \left\{\lambda^{\frac{1}{2}}, \lambda^{\frac{3}{2}}\right\} \max \left\{\left|x_{5}\right|^{\frac{1}{2}},\left|x_{5}\right|^{\frac{1}{3}}\right\}}
$$

which is not very restrictive.

Remark 5. As discussed in Remark 3, there might be many different choices for $\phi(x)$ satisfying relationship (15). Besides the choice made in the paper above, other candidates are also feasible. Indeed, it can be verified that any choice of the form $\phi(x)=x_{5}^{\frac{1}{2 i+1}}$ with $i \geq 1$ will lead to (different) controllers for system (16). Generally speaking, the larger $i$ is, the more computations are involved, and the slower the convergence of the resulting controller is.

Remark 6. Note that feedback controllers for linear system (23) are not limited to form (24). Other choices could also be made, and accordingly, different controllers for chained system (16) could be obtained by following the same procedure discussed above.

Remark 7. In Laiou and Astolfi (1999), the system is divided into the 'base' subsystem and the 'extended' subsystem. The base subsystem is linear and time-invariant, accordingly, linear feedback control laws can stabilize this subsystem. The resulting extended subsystem is linear and time-varying, and a time-varying design technique 
is utilised to present stabilizing feedback laws for it. The proposed controllers yield exponential convergence of the state to the origin. Similar 'base-extended' decomposition technique has also been used in Egeland and Berglund (1994) to develop a time-varying control strategy for system (1) to guarantee asymptotic stability with exponential convergence. In this paper, a new approach is based on nonregular feedback linearization of the whole system, thus it involves neither system division nor time-varying design technique. As the approaches are essentially different, the resulted controllers differ from each other accordingly.

\section{Stabilization of high-order general chained systems}

The design mechanism presented in Section 3 can be extended to the more general class of nonholonomic systems (14). The design procedure is outlined as follows.

Let the first control be

$$
u_{1}=\phi(x)= \begin{cases}y_{l^{\frac{1}{n}}} & \text { if } n \text { is odd } \\ y_{l^{\frac{1}{n+1}}} & \text { if } n \text { is even }\end{cases}
$$

Denote the first-order state-space description of system (14) as

$$
\dot{x}=f(x)+g_{1}(x) u_{1}+g_{2}(x) u_{2}, \quad x \in \Re^{n} .
$$

Let $f_{0}(x)=f(x)+g_{1}(x) \phi(x)$, and $g_{0}(x)=g_{2}(x)$.

Define a real-valued function $h(x)=x_{1}$, and new coordinates $z$ and input $v$ as

$$
\begin{aligned}
& z=T(x)=\left[h, L_{f_{0}} h, L_{f_{0}}^{2} h, \cdots, L_{f_{0}}^{n-1} h\right]^{T} \\
& v=L_{f_{0}}^{n} h+u_{2} L_{g_{0}} L_{f_{0}}^{n-1} h
\end{aligned}
$$

The explicit expressions for $z$ and $v$ can be calculated routinely, though they become more and more tedious as $n$ increasing. Note that $T(x)$ is discontinuous on the surface $S_{D}^{n}=\left\{x \in \Re^{n}: y_{l}=0\right\}$.

It may be verified that the inverse of $z=T(x)$, denoted by $x=T^{-1}(z)$, is a vector function with polynomial components. Therefore, exponential convergence of $z(t)$ implies exponential convergence of $x(t)=T^{-1}(z(t))$. 
Consider the Brunovsky canonical system (12). It is standard that we may find a linear feedback control

$$
v=-K v=-\sum_{i=1}^{n} k_{i} z_{i}
$$

to exponentially stabilize this system. Let $W$ denote the subset of $\Re^{n}$ such that every trajectory of the closed-loop system started from $W$ will never cross the surface $S_{D}^{n}$. Define $D_{n}=\left\{x \in \Re^{n}: T(x) \in W\right\}$. For nonholonomic system (14) with control law

$$
\left\{\begin{array}{l}
u_{1}=\phi(x) \\
u_{2}=-\left(L_{g_{0}} L_{f_{0}}^{n-1} h\right)^{-1}\left(L_{f_{0}}^{n} h(x)+K T(x)\right)
\end{array}\right.
$$

every trajectory of the closed-loop system started within $D_{n}$ will exponentially approach to the origin.

To achieve global stabilization, before applying control law (29), a control strategy must be exploited to drive an arbitrary configuration outside $D_{n}$ into $D_{n}$ in finite time. This strategy may depend on the parameters $k_{i}, i=1, \cdots, n$ and could be determined accordingly.

\section{Simulation studies}

In this section, simulation studies are carried out to demonstrate the effectiveness and procedure of the proposed approach.

Consider a PPR robot moving on a horizontal plain. All the three joints are passive and the only available inputs are forces acting on the end-effector. Let $m_{i}$ be the mass of the $i$ th link, $d_{3}$ the distance between the center of mass of the third link and the third joint axis, $l_{3}$ the length of the third link, and $\mathcal{I}_{3}$ the central moment of inertia of the third link.

The dynamic model of the robot is (De Luca, Mattone and Oriolo 1998)

$$
M\left(q_{3}\right) \ddot{q}+H\left(q_{3}, \dot{q}_{3}\right)=J^{T}\left(q_{3}\right) F
$$

where $q_{i}, i=1,2,3$ are the generalised coordinates, $F=\left[F_{x}, F_{y}\right]^{T}$ are the Cartesian 
forces acting on the end-effector, and

$$
\begin{aligned}
M\left(q_{3}\right) & =\left[\begin{array}{ccc}
a_{1} & 0 & a_{4} \cos q_{3} \\
0 & a_{2} & -a_{4} \sin q_{3} \\
a_{4} \cos q_{3} & -a_{4} \sin q_{3} & -a_{3}
\end{array}\right] \\
H\left(q_{3}, \dot{q}_{3}\right) & =-a_{4} \dot{q}_{3}^{2}\left[\begin{array}{c}
\sin q_{3} \\
\cos q_{3} \\
0
\end{array}\right] \\
J\left(q_{3}\right) & =\left[\begin{array}{ccc}
0 & 1 & -l_{3} \sin q_{3} \\
1 & 0 & l_{3} \cos q_{3}
\end{array}\right]
\end{aligned}
$$

with

$$
\begin{aligned}
& a_{1}=m_{1}+m_{2}+m_{3} \\
& a_{2}=m_{2}+m_{3} \\
& a_{3}=\mathcal{I}_{3}+m_{3} d_{3}^{2} \\
& a_{4}=m_{3} d_{3}
\end{aligned}
$$

Under the condition that $q_{3} \neq \frac{\pi}{2}+k \pi, k=0, \pm 1, \pm 2, \cdots$, the dynamic equations can be transformed into the second-order Caplygin form (De Luca, Mattone and Oriolo 1998)

$$
\begin{aligned}
& \ddot{q}_{2}=v_{1} \\
& \ddot{q}_{3}=v_{2} \\
& \ddot{q}_{1}=a_{5} \tan q_{3} v_{1}+a_{6} \sec q_{3} v_{2}
\end{aligned}
$$

where

$$
\begin{aligned}
& v_{1}=\frac{\left(a_{4}^{2}-a_{1} a_{3}\right) a_{4} c_{3} \dot{q}_{3}^{2}}{\rho_{1}}+\frac{a_{4} a_{1} l_{3} s_{3}^{2}+a_{4}^{2} c_{3}^{2}-a_{1} a_{3}}{\rho_{1}} F_{x}+\frac{\left(a_{4}-a_{1} l_{3}\right) a_{4} s_{3} c_{3}}{\rho_{1}} F_{y} \\
& v_{2}=\frac{\left.a_{2}-a_{1}\right) a_{4}^{2} s_{3} c_{3} \dot{q}_{3}^{2}}{\rho_{2}}+\frac{\left(a_{2} l_{3}-a_{4}\right) a_{1} s_{3}}{\rho_{2}} F_{x}+\frac{\left(a_{4}-a_{1} l_{3}\right) a_{2} c_{3}}{\rho_{2}} F_{y} \\
& a_{5}=\frac{a_{4}-a_{2} l_{3}}{a_{4}-a_{1} l_{3}}, \quad a_{6}=\frac{a_{4} l_{3}-a_{3}}{a_{4}-a_{1} l_{3}}, s_{3}=\sin q_{3}, \quad c_{3}=\cos q_{3} \\
& \rho_{1}=-a_{1} a_{2} a_{3}+a_{1} a_{4}^{2}-a_{1} a_{4}^{2} c_{3}^{2}+a_{4}^{2} a_{2} c_{3}^{2}, \rho_{2}=-a_{1} a_{2} a_{3}+a_{1} a_{4}^{2} s_{3}^{2}+a_{2} a_{4}^{2} c_{3}^{2}
\end{aligned}
$$


System (31) can be converted to form (1) by the following state and input transformations

$$
\begin{aligned}
& \left\{\begin{array}{l}
y_{1}=q_{2}-\frac{a_{6}}{a_{5}}\left(\cos q_{3}-1\right) \\
y_{2}=a_{5} \tan q_{3} \\
y_{3}=q_{1}-a_{6} \sin q_{3}
\end{array}\right. \\
& \left\{\begin{array}{l}
u_{1}=v_{1}+\frac{a_{6}}{a_{5}} \sin q_{3} v_{2}+\frac{a_{6}}{a_{5}} \cos q_{3} \dot{q}_{3}^{2} \\
u_{2}=a_{5} \sec ^{2} q_{3} v_{2}+2 a_{5} \sec ^{2} q_{3} \tan q_{3} \dot{q}_{3}^{2}
\end{array}\right.
\end{aligned}
$$

In summary, the original PPR robot model (30) can be transformed into system (1) by state transformation (32) and input transformation

$$
\left\{\begin{array}{l}
u_{1}=\frac{\left(a_{4}^{2}-a_{1} a_{3}\right) a_{7} c_{3} \dot{q}_{3}^{2}}{a_{5} \rho_{2}}+\frac{\left(a_{4}^{2} c_{3}^{2}-a_{1} a_{3}\right) a_{5}+a_{1} a_{9} s_{3}^{2}}{a_{5} \rho_{2}} F_{x}+\frac{s_{3} c_{3} a_{7} a_{4}-a_{1} l_{3}}{a_{5} \rho_{2}} F_{y} \\
u_{2}=\frac{\sec ^{2} q_{3} a_{5} a_{4}^{2} c_{3} s_{3}\left(a_{2}-a_{1}\right) \dot{q}_{3}^{2}}{\rho_{2}}+2 a_{5} \sec ^{2} q_{3} \tan q_{3} \dot{q}_{3}^{2}+\frac{a_{1} a_{5} a_{8} s_{3}}{\rho_{2}} F_{x}+\frac{a_{2} c_{3}}{\rho_{2}} F_{y}
\end{array}\right.
$$

where

$$
a_{7}=a_{2} a_{6}+a_{4} a_{5}, a_{8}=\sec ^{2} q_{3}\left(a_{2} l_{3}-a_{4}\right), a_{9}=\left(a_{2} a_{6} l_{3}+a_{4} a_{5} l_{3}-a_{4} a_{6}\right)
$$

The values of the parameters $m_{i}, i=1,2,3$ and $\mathcal{I}, l$ in this simulation are shown in Table 1.

Table 1. Parameters used for simulation

\begin{tabular}{ll}
\hline$\left[m_{1}, m_{2}, m_{3}\right]$ & {$[0.5,0.5,1.0] \mathrm{kg}$} \\
$\mathcal{I}_{3}$ & $1 \mathrm{~kg} \mathrm{~m}^{2}$ \\
{$\left[d_{3}, l_{3}\right]$} & {$[1,2] \mathrm{m}$} \\
\hline
\end{tabular}

Suppose the joint configuration is initially at

$$
q_{0}=(0.93,5.43,-0.40 \pi)[\mathrm{m}, \mathrm{m}, \mathrm{rad}], \quad \dot{q}_{0}=(0,2.50,0)[\mathrm{m} / \mathrm{s}, \mathrm{m} / \mathrm{s}, \mathrm{rad} / \mathrm{s}]
$$

The final desired state is the equilibrium

$$
q_{d}=\dot{q}_{d}=[0,0,0]^{T}
$$


The corresponding initial state for system (1) is computed to be

$$
x_{0}=[5.50,-2.50,-2.20,0.00,1.00,0.00]^{T} \in D
$$

Accordingly, pure state feedback controller (25) can be used. Let $\lambda=1$. Figures 1 and 2 show the convergence of the generalised coordinates and velocities, while figure 3 demonstrates the boundedness of the applied forces. As shown in figure 1, the angle $q_{3}$ never crosses the singular points $\pm \frac{\pi}{2}$.

If the joint configuration is initially at

$$
q_{0}=\left(5,0, \frac{\pi}{4}\right)[\mathrm{m}, \mathrm{m}, \mathrm{rad}], \quad \dot{q}_{0}=(0,0,0)[\mathrm{m} / \mathrm{s}, \mathrm{m} / \mathrm{s}, \mathrm{rad} / \mathrm{s}]
$$

Then the corresponding initial state for system (1) is

$$
x_{0}=[0.029,0,0.714,0,4.949,0]^{T} \notin D
$$

Because the initial state is outside set $D$, appropriate control laws have to be conducted to drive the system into $D$ in a finite time, then the discontinuous controller is applicable. Figures 4 and 5 shows the converging trajectories of the generalised coordinates and velocities, respectively. Figure 6 demonstrates the boundedness of the applied forces. Due to the switching of the control signals, the trajectories are not smooth as shown in figures 4 and 5 .

\section{Conclusion}

In this paper, a new nonregular feedback linearization mechanism has been proposed to steer the second-order nonholonomic chained systems approaching the origin with an exponential rate of convergence. A sufficient condition for nonsmooth nonregular feedback linearization was presented for affine nonlinear systems with two inputs. It has been proven that the second-order nonholonomic chained systems are nonregular static state feedback linearizable. A discontinuous control law was designed based on linear system theory and the inversion technique. To globally stabilize the systems, a logic-based switching strategy among different control schemes was developed. A simulation study has also been provided to show the effectiveness of the control scheme. 


\section{References}

Arai, H., Tanie, K., and Shiroma, N., 1997, Feedback control of 3-DOF planar underactuated manipulator. Proceedings of the IEEE Conference on Robotics and Automation, pp. 703-709.

Astolfi, A., 1996, Discontinuous control of nonholonomic systems. Systems and Control Letters, 27, 37-45.

Bhat, S. P., and Bernstein, D. S., 1998, Continuous finite-time stabilization of the translational and rotational double integrators. IEEE Transactions on Automatic Control, 43, 678-682.

Canudas de Wit, C., Siciliano, B., and Bastin, G., 1996, Theory of Robot Control (London: Spinger).

Charlet, B., Levine, J., and Marino, R., 1989, On dynamic feedback linearization. Systems and Control Letters, 13, 143-151.

Chiasson, J., 1998, A new approach to dynamic feedback linearization control of an induction motor. IEEE Transactions on Automatic Control, 43, 391-397.

Egeland, O., and Berglund, E., 1994, Control of an underwater vehicle with nonholonomic acceleration constraints. IFAC Conference on Robot Control, Capri, Italy, pp. 845-850.

Ge, S. S., Lee, T. H., and Harris, C. J., 1998, Adaptive Neural Network Control of Robotic Manipulators (NJ : World Scientific).

Kolmanovsky, I., and McClamroch, N. H., 1995, Developments in nonholonomic control problems. IEEE Control Systems, 15(6), 20-36.

Laiou, M., and Astolfi, A., 1999, Discontinuous control of high-order generalized chained systems. Systems and Control Letters, 37, 309-322. 
De Luca, A., Mattone, R., and Oriolo, G., 1996, Dynamic mobility of redundant robots using end-effector commands. Proceedings of the IEEE Conference on Robotics and Automation, pp. 1760-1767.

De Luca, A., Mattone, R., and Oriolo, G., 1998, Steering a class of redundant mechanisms through end-effector generalized forces. IEEE Transactions on Robotics and Automation, 14, 329-333.

Luo, J., and Tsiotras, P., 2000, Control design for chained-form systems with bounded inputs. Systems and Control Letters, 39, 123-131.

M'Closkey, R., and Morin, P., 1998, Time-varying homogeneous feedback: design tools for the exponential stabilization of systems with drift. International Journal of Control, 71, 837-869.

Murray, R. M., Li, Z., and Sastry, S. S., 1994, A Mathematical Introduction to Robotic Manipulation (London: CRC press).

Oriolo, G., and Nakamura, Y., 1991, Control of mechanical systems with secondorder nonholonomic constraints: underactuated manipulators. Proceedings of the IEEE Conference on Decision and Control, pp. 306-308.

Reyhanoglu, M., van der Schaft, A., McClamroch, N. H., and Kolmanovsky, I., 1999, Dynamics and control of a class of underactuated mechanical systems. IEEE Transactions on Automatic Control, 44, 1663-1671.

Seto, D., and Baillieul, J., 1994, Control problems in super-articulated mechanical systems. IEEE Transactions on Automatic Control, 39, 2442-2453.

Sordalen, O. J., 1993, Feedback Control of Nonholonomic Mobile Robots, Doctoral dissertation, Department of Engineering Cybernetics, The Norwegian Institute of Technology.

Spong, M. W., 1995, The swing up control problem for the acrobat. IEEE Control Systems, 15(6), 49-55. 
Spong, M. W., and Vidyasagar, M., 1989, Robot Dynamics and Control (New York: Wiley).

Su, C., and Stepanenko, Y., 1999, Adaptive variable structure set-point control of underactuated robots. IEEE Transactions on Automatic Control, 44, 2090-2093.

Sun, Z., and Xia, X., 1997, On nonregular feedback linearization. Automatica, 33, $1339-1344$.

Taylor, D. G., 1994, Nonlinear control of electric machines: an overview. IEEE Control Systems, 14(4), 41-51.

Wichlund, K., Sordalen, O. J., and Egeland, O., 1995, Control of vehicles with second-order nonholonomic constrains: underactuated vehicles. Proceedings of European Control Conference, Rome, Italy, pp. 3086-3091. 


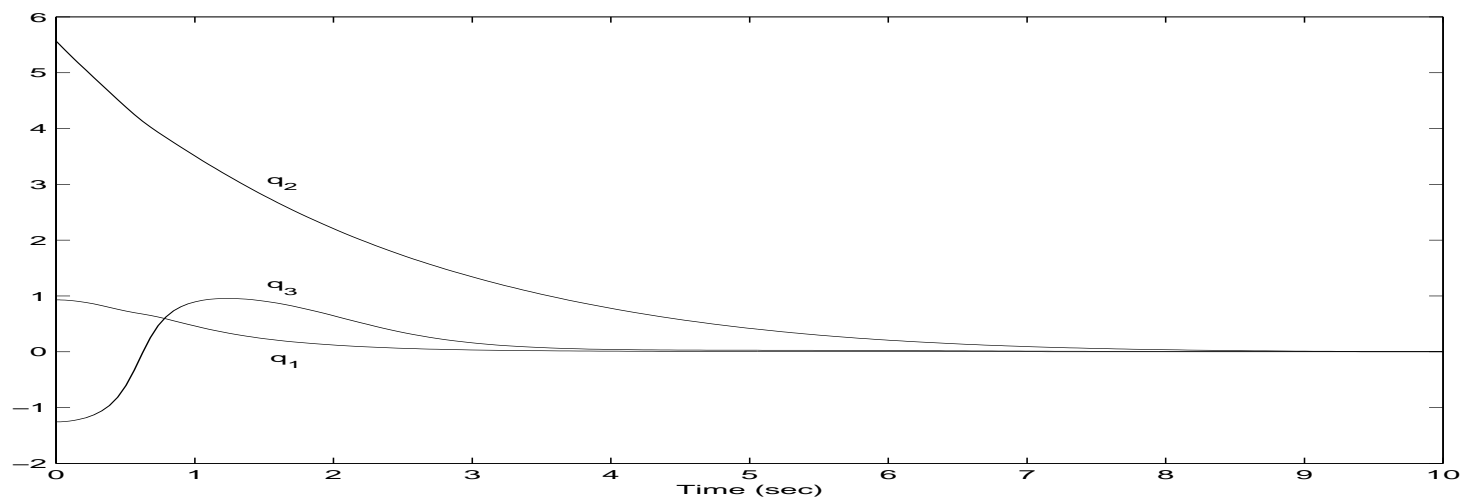

Figure 1: The generalised coordinates with continuous forces

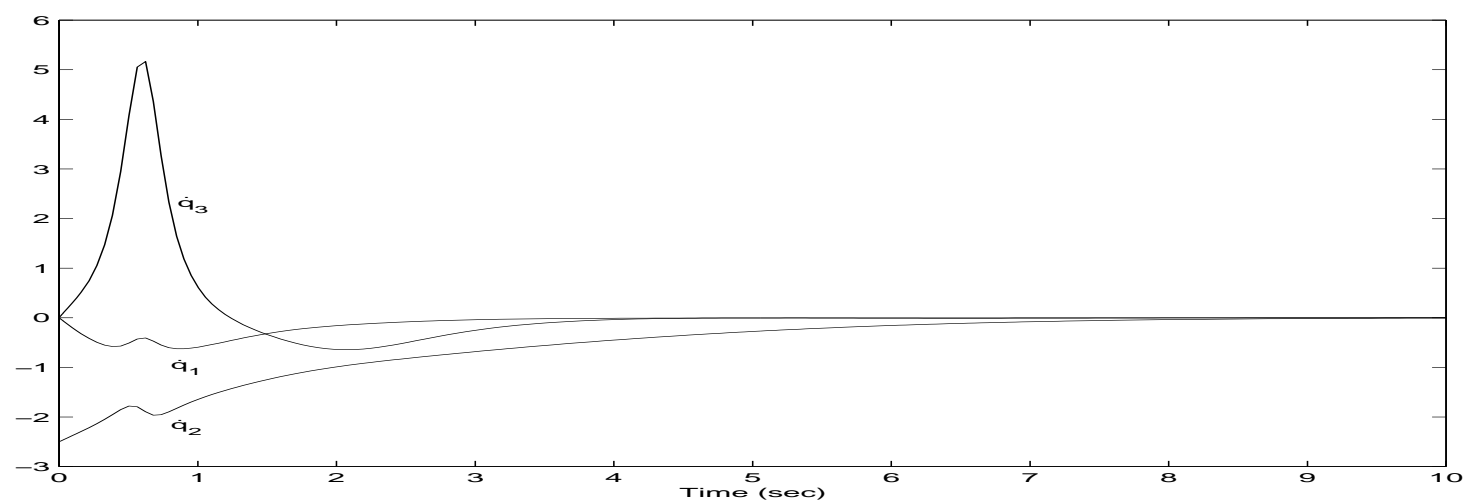

Figure 2: The generalised velocities with continuous forces 


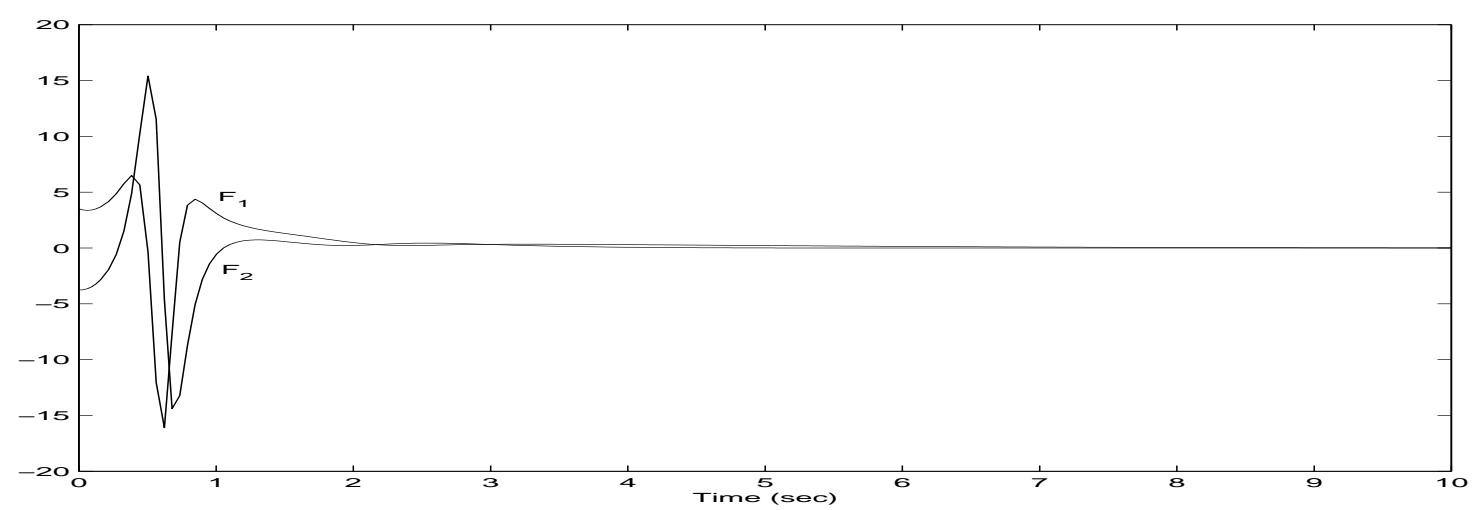

Figure 3: The applied forces

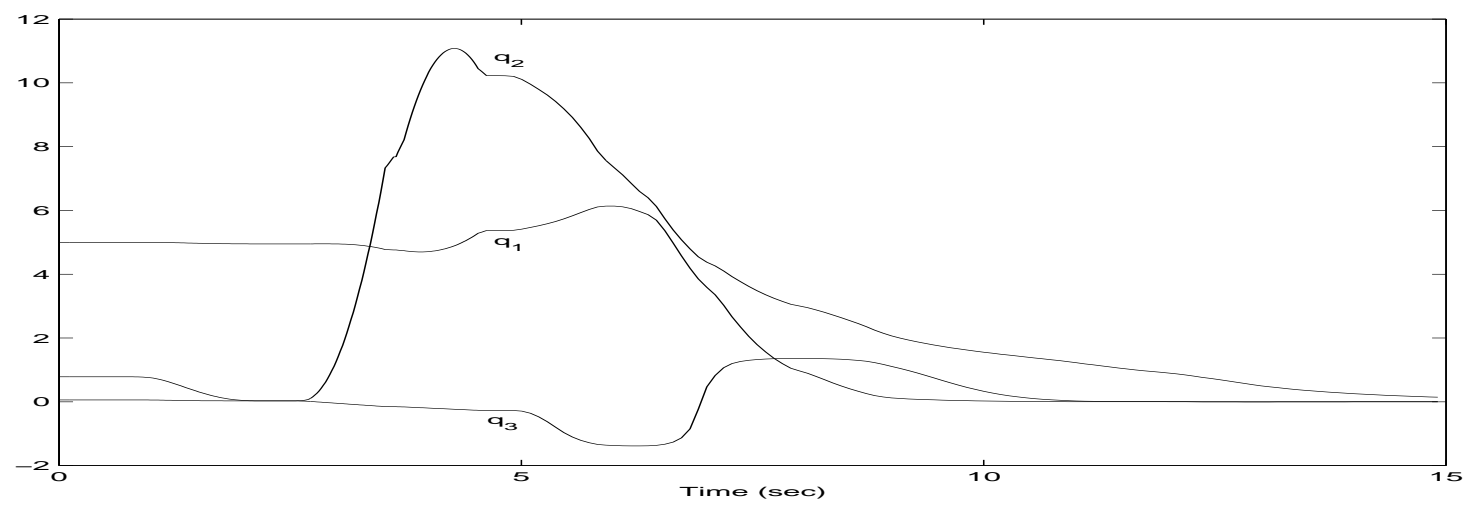

Figure 4: The generalised coordinates with discontinuous forces

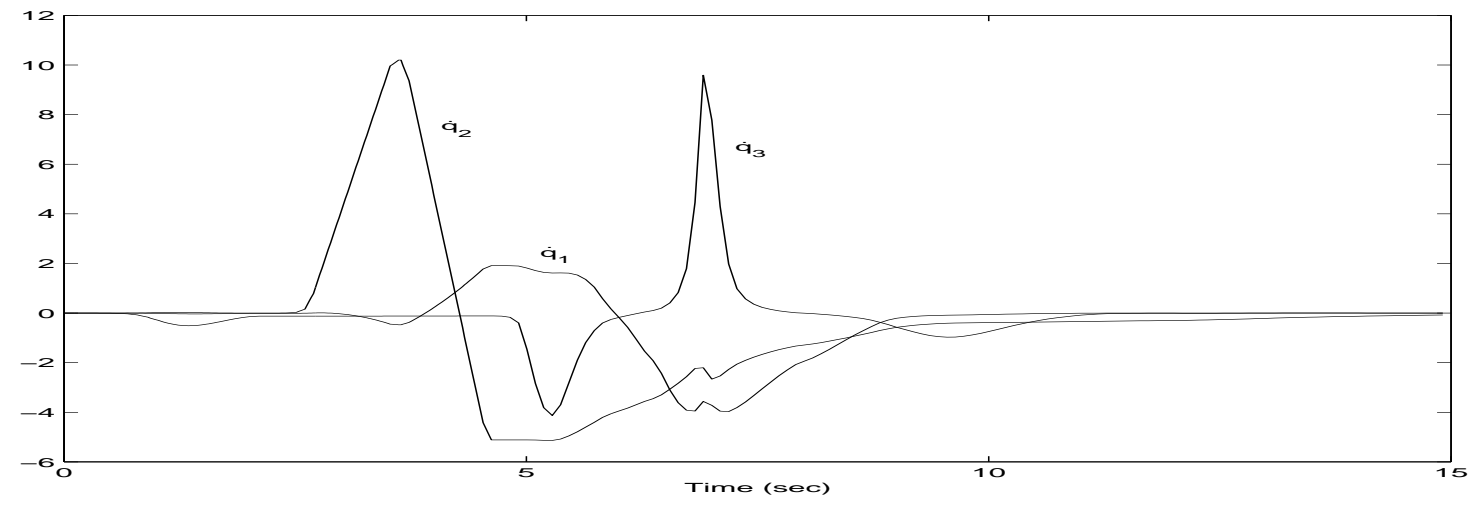

Figure 5: The generalised velocities with discontinuous forces 


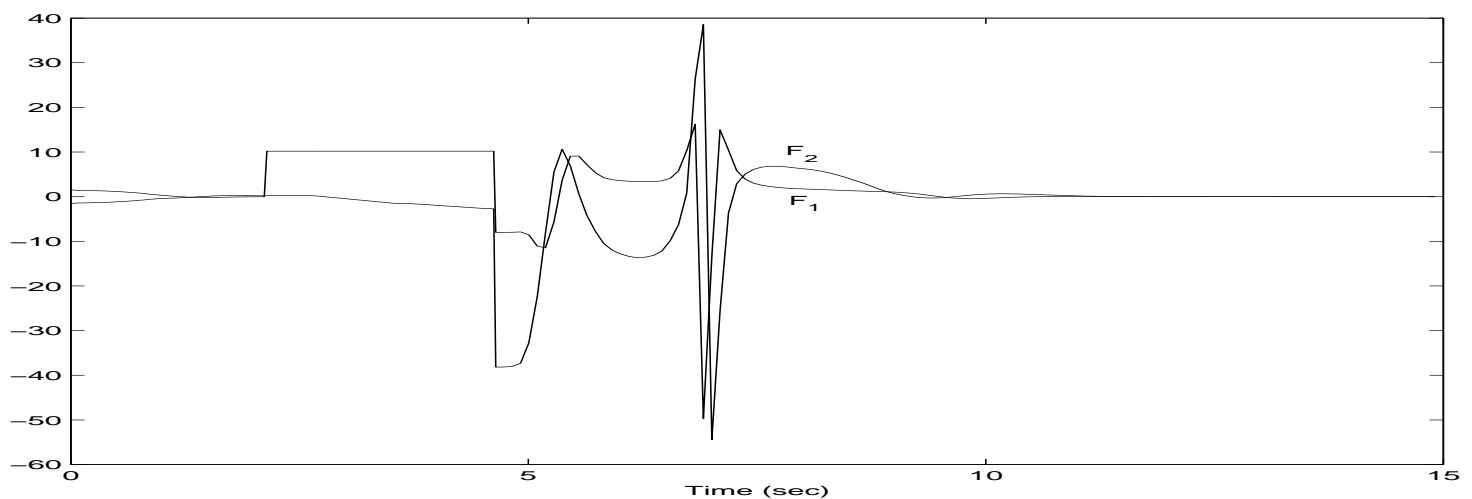

Figure 6: Trajectories of discontinuous forces 